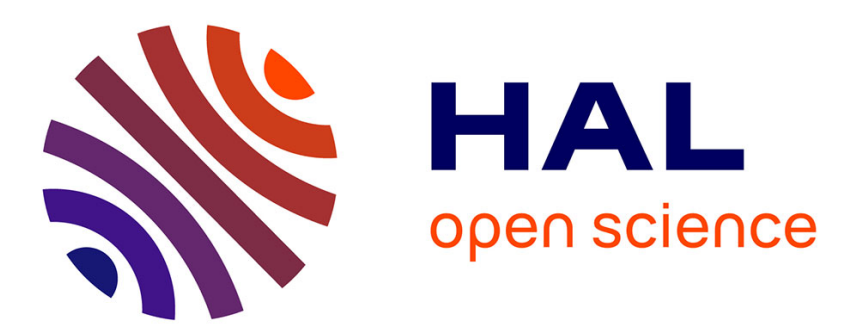

\title{
Convex shapes and convergence speed of discrete tangent estimators
}

Jacques-Olivier Lachaud, François de Vieilleville

\section{To cite this version:}

Jacques-Olivier Lachaud, François de Vieilleville. Convex shapes and convergence speed of discrete tangent estimators. Proc. Int. Symposium on Visual Computing (ISVC2006), Nov 2006, Lake Tahoe, Nevada, United States. pp.688-697, 10.1007/11919629 . hal-00308337

\section{HAL Id: hal-00308337 https://hal.science/hal-00308337}

Submitted on 17 Jun 2009

HAL is a multi-disciplinary open access archive for the deposit and dissemination of scientific research documents, whether they are published or not. The documents may come from teaching and research institutions in France or abroad, or from public or private research centers.
L'archive ouverte pluridisciplinaire HAL, est destinée au dépôt et à la diffusion de documents scientifiques de niveau recherche, publiés ou non, émanant des établissements d'enseignement et de recherche français ou étrangers, des laboratoires publics ou privés. 


\title{
Convex shapes and convergence speed of discrete tangent estimators
}

\author{
Jacques-Olivier Lachaud and François de Vieilleville
}

LaBRI, Univ. Bordeaux 1, 351 cours de la Libération, 33405 Talence Cedex, France

\begin{abstract}
Discrete geometric estimators aim at estimating geometric characteristics of a shape with only its digitization as input data. Such an estimator is multigrid convergent when its estimates tend toward the geometric characteristics of the shape as the digitization step $h$ tends toward 0 . This paper studies the multigrid convergence of tangent estimators based on maximal digital straight segment recognition. We show that such estimators are multigrid convergent for some family of convex shapes and that their speed of convergence is on average $\mathcal{O}\left(h^{\frac{2}{3}}\right)$. Experiments confirm this result and suggest that the bound is tight.
\end{abstract}

\section{Introduction}

The problem of estimating geometric quantities of digitized shapes like area, perimeter, tangents or curvatures is an important and active research field. Discrete geometric estimators have indeed many applications in vision, shape analysis and pattern recognition. It is however difficult to compare objectively their respective accuracy, since for a given shape there exists infinitely many shapes with the same digitization. In this paper, we are mainly interested by the multigrid convergence property of some estimators, which is one of the few existing objective criteria. This property ensures that a better resolution brings a better approximation. For concrete applications, the speed of convergence is a very important criterion too, since it has a huge impact on their accuracy at standard resolutions.

Formally, taking the definitions of [9], let $\operatorname{Dig}_{h}$ be some digitization process of step $h$. Let $\mathbb{F}$ be a family of shapes in $\mathbb{R}^{2}$ and let $G$ be a geometric quantity defined for all $X \in \mathbb{F}$. A discrete estimator of $G$ is a map that associates to a digitization $\operatorname{Dig}_{h}(X)$ an estimation of $G(X)$. A discrete estimator $\mathcal{G}$ is multigrid convergent toward $G$ for $\mathbb{F}$ and Dig iff, for any $X \in \mathbb{F}$, there exists some $h_{X}>0$ for which

$$
\forall 0<h<h_{X},\left|\mathcal{G}\left(\operatorname{Dig}_{h}(X)\right)-G(X)\right| \leq \tau(h),
$$

where $\tau: \mathbb{R}^{+} \rightarrow \mathbb{R}^{+*}$ has limit value 0 at $h=0$. This function defines the speed of convergence of $\mathcal{G}$ toward $G$.

For instance, for the family of plane convex bodies with $C^{3}$-boundary and positive curvatures, denoted later on by $\mathbb{F}_{c}^{3}$, the best known speed of convergence for an area and or a moment estimator is $\mathcal{O}\left(h^{\frac{15}{11}}\right)$ [8, 10], for a perimeter estimator it is $\mathcal{O}(h)$ [16]. There are fewer results concerning local geometric quantities like 
tangent direction or curvature. The first works on this topic were presented in [4], where some evidence of the convergence of tangent estimators based on digital straight segment (DSS) recognition were given. We report the recent work of [13 which establishes the multigrid convergence of tangent direction estimators based on maximal DSS recognition, with an average speed of convergence of $\mathcal{O}\left(h^{\frac{1}{3}}\right)$. The recent result of [5] has confirmed that there is yet no curvature estimator proven to be multigrid convergent.

In this paper, we prove a new upper bound for the average speed of convergence of discrete tangent estimators which are based on maximal DSS recognition around the point of interest [6, 12]. This new bound of $\mathcal{O}\left(h^{\frac{2}{3}}\right)$ was suggested by the experimental study of 12 and enhances the previous bound of $\mathcal{O}\left(h^{\frac{1}{3}}\right)[13$. The proof of this enhanced result, obtained for shapes in $\mathbb{F}_{c}^{3}$, follows these steps:

Section 2 Digitizations of convex shapes are digital convex polygons (CDP). We achieve thus a better localization of the shape boundary with respect to the digitized boundary (Proposition 11).

Section 3 We first claim that the DSS characteristics have an average asymptotic behaviour depending on their length (Claim 2). The asymptotic edge length of CDP is recalled (Theorem 1), which induces a superlinear localization of the shape boundary in $\mathcal{O}\left(h^{\frac{4}{3}}\right)$ (Proposition 2). With these relations, the slope of digital edges of $\mathrm{CDP}$ is shown to be multigrid convergent to the tangent direction of a nearby boundary point with an average speed of $\mathcal{O}\left(h^{\frac{2}{3}}\right)$ (Proposition 3).

Section 4 The behaviour of maximal DSS is identical to the behavior of digital edges, since any maximal DSS contains at least one digital edge (Proposition 4 ). The average speed of convergence of tangent estimators based on maximal DSS is thus achieved (Theorem 3).

We will then conclude and open some perspectives in Section 5.

\section{Preliminary definitions and first properties}

\subsection{Digitization and convex digital polygon}

Let $S$ be some subset of $\mathbb{R}^{2}$. Its Gauss digitization of grid step $h$ is defined as $\mathrm{D}_{h}^{G}(S)=S \cap h \mathbb{Z} \times h \mathbb{Z}$. Thus, the considered digitized objects are subsets of the rescaled digital plane $h \mathbb{Z} \times h \mathbb{Z}$.

A convex digital polygon (CDP) $\Gamma$ is a subset of the digital plane $h \mathbb{Z} \times h \mathbb{Z}$ with a single 4-connected component equal to the Gauss digitization of its convex hull, i.e. $\Gamma=\mathrm{D}_{h}^{G}(\operatorname{conv}(\Gamma))$. Its vertices $\left(V_{i}\right)_{i=1 . . e}$ form the minimal subset for which $\Gamma=\mathrm{D}_{h}^{G}\left(\operatorname{conv}\left(V_{1}, \ldots, V_{e}\right)\right)$ (vertices are ordered clockwise). The points of $\Gamma$ which are 8-adjacent to some point not in $\Gamma$ form the border of $\Gamma$. It is a 4-connected digital path that visits every $V_{i}$ in order. When moving clockwise, any subpath $\Gamma_{V_{i}, V_{i+1}}$ is called a digital edge of $\Gamma$, while the Euclidean straight segment $V_{i} V_{i+1}$ is an edge of $\Gamma$.

For small enough grid steps, Gauss digitizations of any shape in $\mathbb{F}_{c}^{3}$ are convex digital polygons. This result will be admitted throughout the paper. 


\subsection{Standard line, digital straight segment, maximal segments}

Definition 1. 15] The set of points $(x, y)$ of the digital plane $\mathbb{Z}^{2}$ verifying $\mu \leq$ $a x-b y<\mu+|a|+|b|$, with $a, b$ and $\mu$ integer numbers, is called the standard line with slope $a / b$ and shift $\mu$. Standard lines are the 4 -connected discrete lines.

The quantity $a x-b y$ is called the remainder of the line. Points whose remainder is $\mu$ (resp. $\mu+|a|+|b|-1$ ) are called upper (resp. lower) leaning points. Any finite connected portion of a standard line is called a digital straight segment (DSS). Its characteristics are the slope $a / b$ and the shift $\mu$ of the standard line containing it with smallest $|a|+|b|$.

Most of the results demonstrated here are directly transferable for 8-connected curves since there is a natural bijective transformation between standard and naive digital lines. In the paper, all the reasoning is made in the first octant, but it extends naturally to the whole digital plane.

\subsection{Recursive decomposition of DSS}

We here recall a few properties about patterns composing DSS and their close relations with continued fractions. They constitute a powerful tool to describe discrete lines with rational slopes [2,7]. Without loss of generality all definitions and propositions stated below hold for standard lines and DSS with slopes in the first octant (e.g. $\frac{a}{b}$ with $0 \leq a \leq b$ ). In the first octant, only two Freeman moves are possible, 0 is a step to the right and 1 is a step up, 4-connected digital paths can be expressed as words of $\{0,1\}^{*}$.

Definition 2. Given a standard line $(a, b, \mu)$, we call pattern of characteristics $(a, b)$ the word that is the succession of Freeman moves between any two consecutive upper leaning points. The Freeman moves defined between any two consecutive lower leaning points is the previous word read from back to front and is called the reversed pattern.

Since a DSS has at least either two upper or two lower leaning points, a DSS $(a, b, \mu)$ contains at least one pattern or one reversed pattern of characteristics $(a, b)$. There exists recursive transformations for computing the pattern of a standard line from the simple continued fraction of its slope (see [2], [18] Chap. 4 and [9] Chap. 9), here Berstel approach better suits our purpose.

A rational slope $z$ in $] 0,1]$ can be written uniquely as the continued fraction

$$
z=0+\frac{1}{u_{1}+\frac{1}{\ldots+\frac{1}{u_{n}}}}
$$

and is conveniently denoted $\left[0, u_{1}, \ldots, u_{n}\right]$. The $u_{i}$ are called the partial coefficients and the continued fraction formed with the $k$ first partial coefficient is said to be a $k$-th convergent of $z$ and is a rational numbers denoted by $z_{k}$. The 


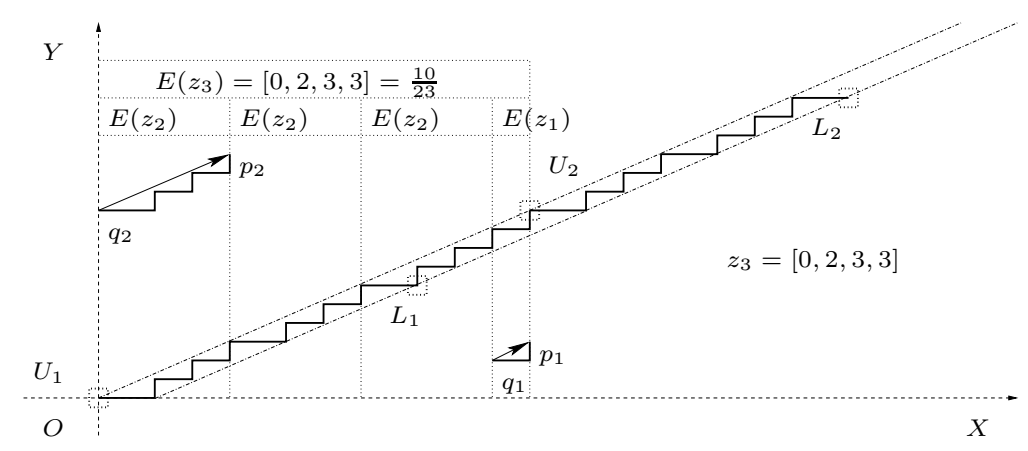

Fig. 1. A digital straight segment of characteristics $(10,23,0)$ with an odd depth slope, taken between the origin and its second lower leaning point

depth of a $k$-th convergent equals $k$. We conveniently denote $p_{k}$ the numerator (resp. $q_{k}$ the denominator) of a $k$-th convergent.

We recall a few more relations regarding the way convergents are related and which will be used later on in this paper:

$$
\begin{array}{llll} 
& & \forall k \geq 1 & p_{k} q_{k-1}-p_{k-1} q_{k}=(-1)^{k+1} \\
p_{0}=0 & p_{-1}=1 & \forall k \geq 1 & p_{k}=u_{k} p_{k-1}+p_{k-2} \\
q_{0}=1 & q_{-1}=0 & \forall k \geq 1 & q_{k}=u_{k} q_{k-1}+q_{k-2}
\end{array}
$$

Given a rational slope between 0 and 1 its continued fraction is finite and for each $i, u_{i}$ is a strictly positive integer. In order to have a unique writing we consider that the last partial coefficient is greater or equal to two; except for slope $1=[0,1]$.

Let us now explain how to compute the pattern associated with a rational slope $z$ in the first octant. Let us define $E$ a mapping from the set of positive rational number smaller than one onto the Freeman-move's words. More precisely: $E\left(z_{0}\right)=0, E\left(z_{1}\right)=0^{u_{1}} 1$ and the other values are expressed recursively:

$$
\begin{aligned}
E\left(z_{2 i+1}\right) & =E\left(z_{2 i}\right)^{u_{2 i+1}} E\left(z_{2 i-1}\right) \\
E\left(z_{2 i}\right) & =E\left(z_{2 i-2}\right) E\left(z_{2 i-1}\right)^{u_{2 i}}
\end{aligned}
$$

It has been shown that this mapping constructs the pattern $(a, b)$ for any rational slope $z=\frac{a}{b}$. Fig. 1 illustrates the construction of an odd pattern using the mapping $E$. The Minkowski $\mathcal{L}^{1}$ length of $E\left(z_{k}\right)$ equals $p_{k}+q_{k}$ and can be computed recursively using Eq. (2) and (3). Moreover we recall that any digital edge is a pattern or a succession of the same pattern, its digital parameters are its slope, denoted by $\frac{p}{q}$, and the number $\delta$ of repetitions of the pattern $E\left(\frac{p}{q}\right)$.

\subsection{Localization accuracy of digitized convex shapes}

One can expect that the boundary of a convex shape is approximately at distance $h$ from the border of its digitization of grid step $h$. In fact, for a convex shape 


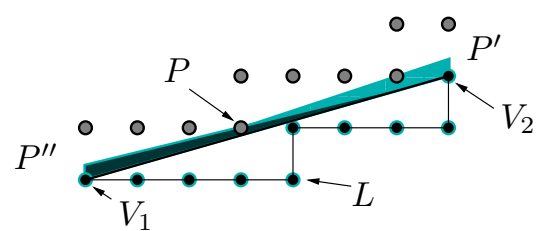

Fig. 2. Two local constraints for the real underlying convex shape

$S$, its boundary $\partial S$ is much closer to the convex hull of $\mathrm{D}_{h}^{G}(S)$ than $h$. A better localization of the shape is thus possible, as stated below and illustrated in Fig. 2:

Proposition 1. Let $S$ be a convex shape such that $\mathrm{D}_{h}^{G}(S)$ is a $C D P \Gamma$ for some $h$. Consider an edge $V_{i} V_{i+1}$ of $\Gamma$ with slope in the first octant. Then any point of the boundary $\partial S$ above the straight segment $V_{i} V_{i+1}$ has a vertical distance to it no greater than $\frac{h}{q_{n-1}}$, where the slope of this edge is the irreducible fraction $\frac{p_{n}}{q_{n}}$.

The proof of this proposition can be found in 11 .

\section{Asymptotic behaviour of edges of digitized shapes}

We study here asymptotic properties of edges of digitized convex shapes. Their average length is first exhibited and an experimental study of the digital parameters of edges is presented. The direction of digital edges is shown to converge toward the tangent direction.

\subsection{Asymptotic number and digital parameters of edges}

Let $S$ be some shape of $\mathbb{F}_{c}^{3}$. We have the following theorem:

Theorem 1. (Adapted from 11], Theorem 2) For a small enough h, the Gauss digitization $\mathrm{D}_{h}^{G}(S)$ of $S$ is a CDP and its number of edges $n_{e}\left(\mathrm{D}_{h}^{G}(S)\right)$ satisfies:

$$
c_{1}(S) \frac{1}{h^{\frac{2}{3}}} \leq n_{e}\left(\mathrm{D}_{h}^{G}(S)\right) \leq c_{2}(S) \frac{1}{h^{\frac{2}{3}}}
$$

where the constants $c_{1}(S)$ and $c_{2}(S)$ depend on extremal bounds of the curvatures along $S$. Hence for a disc $c_{1}$ and $c_{2}$ are absolute constants.

As an immediate corollary, the average Minkowski $\mathcal{L}^{1}$ length of edges grows as $\Theta\left(h^{\frac{2}{3}}\right)$. The question is: what is the average behavior of the digital parameters $p, q$ and $\delta$ of edges, knowing that the average digital length $l=\delta(p+q)$ satisfies $\Theta\left(h^{\frac{2}{3}} / h\right)=\Theta\left(1 / h^{\frac{1}{3}}\right)$ ? Since the slope of edges should tend toward the slope of points on the boundary of $S$ and since almost all these points have irrational slope, $p$ and $q$ should tend toward infinity almost everywhere with a bounded $\delta$. This observation is confirmed by experiments, as illustrated on Fig. 3, which plots the means and standard deviations of $\frac{q}{l}$ and $\delta$ for edges on finer and finer digitizations of a disk. It is clear that $q$ (but also $p$ ) satisfies the same asymptotic law as $l$ while $\delta$ remains bounded on average. We make hence the following claim: 

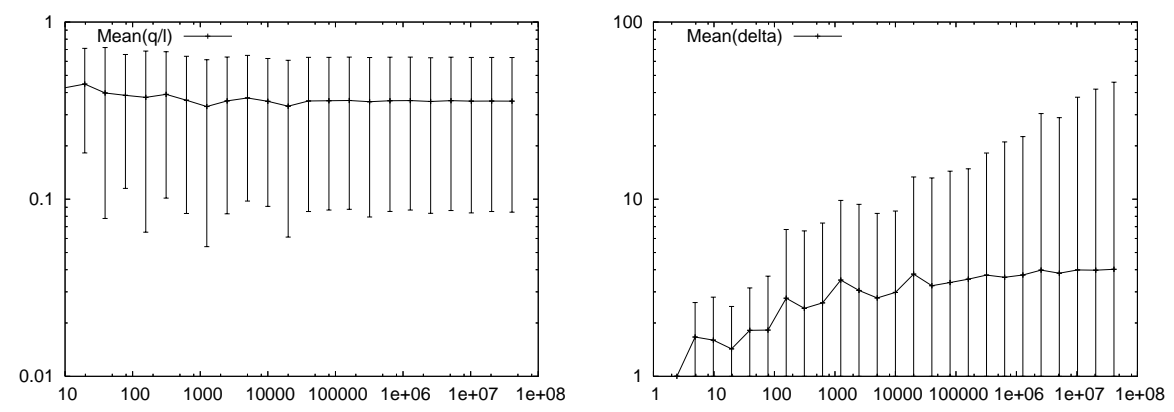

Fig. 3. Plots in log-space of the means of digital parameters $\frac{q}{l}$ (left) and $\delta$ (right) for the edges of Gauss digitizations of a disk, as functions of the inverse $\frac{1}{h}$ of the grid step. Standard deviations are symbolized with errorbars

Claim 2. On average, the digital parameters $p, q$ of the edges of $\mathrm{D}_{h}^{G}(S)$ with $S \in \mathbb{F}_{c}^{3}$ grow as $\Theta\left(\frac{1}{h^{\frac{1}{3}}}\right)$, whereas $\delta$ is bounded, when $h$ tends toward 0 .

This claim induces a first result about the convergence speed of the localization constraints of Proposition 1 ( $\left(q_{n-1}\right.$ has the same asymptotic law as $\left.q_{n}\right)$.

Proposition 2. Let $S \in \mathbb{F}_{c}^{3}$ and $\mathrm{D}_{h}^{G}(S)$ its Gauss digitization. For a small enough $h$, in each octant, the vertical distance between any edge of $\mathrm{D}_{h}^{G}(S)$ and $\partial S$ is bounded. On average, this bound is $\mathcal{O}\left(h^{\frac{4}{3}}\right)$ (and never worse than $\mathcal{O}(h)$ ).

\subsection{Convergence of tangent estimation based on edge direction}

Let $M$ be a point on the boundary of a shape $S \in \mathbb{F}_{c}^{3}$ and let $\theta_{M}(S)$ be the tangent direction of $\partial S$ at $M$. We propose to estimate $\theta_{M}(S)$ as the arctangent of the slope of the digital edge of the $\operatorname{CDP~}_{h}^{G}(S)$ lying below $M$ (for the first octant; definitions for other octants are straightforward). We denote this discrete estimator by $\mathcal{T}_{M}^{\text {edge }}$. Assuming Claim目, we have the multigrid convergence of this tangent estimator:

Proposition 3. Let $M$ be a point in the plane and let $\mathbb{F}_{c}^{3}(M)$ be the subset of shapes $S$ of $\mathbb{F}_{c}^{3}$ with $M \in \partial S$. The discrete estimator $\mathcal{T}_{M}^{\text {edge }}$ at point $M$ is multigrid convergent toward the tangent direction $\theta_{M}$ for $\mathbb{F}_{c}^{3}(M)$ and Gauss digitization. Furthermore, its speed of convergence is on average $\mathcal{O}\left(h^{\frac{2}{3}}\right)$.

Proof. We have to prove that for a shape $S \in \mathbb{F}_{c}^{3}(M)$, there exists some grid step $h_{S}$ for which $\forall 0<h<h_{S},\left|\mathcal{T}_{M}^{\text {edge }}\left(\mathrm{D}_{h}^{G} S\right)-\theta_{M}(S)\right| \leq \tau(h)$. Without loss of generality, we assume that the tangent direction $\theta_{M}(S)$ is in the first octant and we locally parameterize $\partial S$ as $(x, f(x))$, setting $M$ as the origin of the coordinate axes $\left(\left(x_{M}, y_{M}\right)=(0,0)\right)$. Let $h_{0}$ be the digitization step below which $\mathrm{D}_{h}^{G}(S)$ is a CDP. 
Since the slope of $\partial S$ around $M$ is in the first octant, there exists some $h_{S}<h_{0}$ such that for any $0<h<h_{S}$, the vertical straight segment going down from $M$ intersect some edge $V_{i} V_{i+1}$ of $\mathrm{D}_{h}^{G}(S)$. Let $\delta, p$ and $q$ be the digital parameters of this edge, let $z$ be its slope $\frac{p}{q}$ and let $n$ be the depth of $z$ (i.e. $z=\frac{p_{n}}{q_{n}}$ ). The horizontal distance between $M$ and either $V_{i}$ or $V_{i+1}$ is necessarily greater than half the horizontal length of $V_{i} V_{i+1}$. We assume $V_{i+1}$ maximizes this distance, without loss of generality. We get:

$$
\frac{h \delta q_{n}}{2} \leq x_{v_{i+1}} \leq h \delta q_{n}
$$

From Proposition 1 we have for any $x \in\left[x_{v_{i}}, x_{v_{i+1}}\right]$ :

$$
z x-\frac{h}{q_{n-1}} \leq f(x) \leq z x+\frac{h}{q_{n-1}} .
$$

Inserting Taylor expansion of $f(x)$ about $x=0$ in Eq. (7) induces:

$$
\left|z-f^{\prime}(0)\right| \leq \frac{h}{x q_{n-1}}+\mathcal{O}(x) .
$$

Setting $x=x_{v_{i+1}}$ in Eq. (8) and using both sides of Eq. (6) gives the inequality

$$
\left|z-f^{\prime}(0)\right| \leq \frac{2 h}{h \delta q_{n} q_{n-1}}+\mathcal{O}\left(h \delta q_{n}\right) .
$$

We notice that $z=\tan \left(\mathcal{T}_{M}^{\text {edge }}\left(\mathrm{D}_{h}^{G}(S)\right)\right)$ and that $f^{\prime}(0)=\tan \left(\theta_{M}(S)\right)$. Moreover, for any angle $s, t \in\left[0, \frac{\pi}{4}\right]$, we have $|s-t| \leq|\tan (s)-\tan (t)|$. With these two remarks, Eq. (9) implies:

$$
\left|\mathcal{T}_{M}^{\text {edge }}\left(\mathrm{D}_{h}^{G}(S)\right)-\theta_{M}(S)\right| \leq \frac{2}{\delta q_{n} q_{n-1}}+\mathcal{O}\left(h \delta q_{n}\right) .
$$

Since $\delta q_{n}$ is no smaller than half the edge length, it follows from Theorem 1 that the dominant term $\frac{2}{\delta q_{n} q_{n-1}}$ is at least some $\mathcal{O}\left(h^{\frac{1}{3}}\right)$ on average. In 13] it is also shown that there is no edge of bounded length as $h$ tends toward 0 . Then the right part of Eq. (10) tends toward 0. The multigrid convergence is thus shown. At last, assuming Claim 2, Eq. (10) then induces

$$
\left|\mathcal{T}_{M}^{\text {edge }}\left(\mathrm{D}_{h}^{G}(S)\right)-\theta_{M}(S)\right| \leq \mathcal{O}\left(h^{\frac{2}{3}}\right),
$$

which indicates that the average speed of convergence of $\mathcal{T}_{M}^{\text {edge }}$ is $\mathcal{O}\left(h^{\frac{2}{3}}\right)$.

\section{Tangent estimators based on maximal DSS recognition}

This section discusses the convergence speed of discrete tangent estimators based on maximal digital straight segment (maximal segment) recognition. Along a digital path, maximal segments are the inextensible digital straight segments, otherwise said adding the next point to the front or to the back constructs a set of digital points that no standard line contains. The set of all maximal segments of a digital path can be extracted efficiently in time linear with its number of points [6. Maximal segments have deep links with edges of convex hulls [5]. 

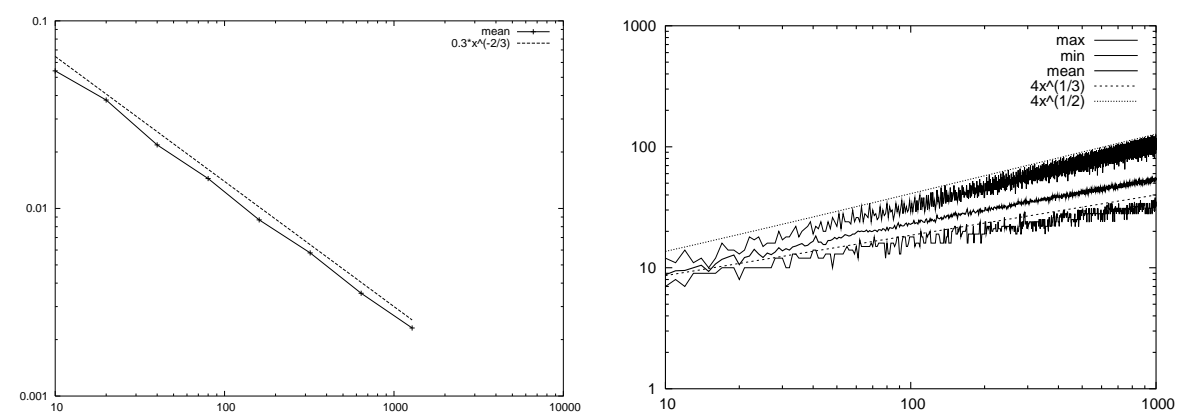

Fig. 4. For both plots, the digitized shape is a disk of radius 1 and the abscissa is the inverse of the digitization step. Left: plot in log-space of the mean absolute error between the $\lambda$-MST tangent direction and the theoretical one (mean taken over all digital points). The convergence speed on this shape is likely to be in $\Theta\left(h^{\frac{2}{3}}\right)$. Right: plot in log-space of the digital length of maximal segments, which is on average $\Theta\left(1 / h^{\frac{1}{3}}\right)$

Estimating the tangent direction at some point is then achieved by considering specific DSS [17] or maximal segments [6] containing this point. A recent experimental evaluation [12] has shown that tangent estimators based on maximal segments are accurate and preserve convexity properties of the real shape.

Let $M$ be a point on the boundary of a shape $S \in \mathbb{F}_{c}^{3}$ and let $\theta_{M}(S)$ be the tangent direction of $\partial S$ at $M$. We propose to estimate $\theta_{M}(S)$ as the arctangent of the slope of any maximal segment of the $\mathrm{CDP} \mathrm{D}_{h}^{G}(S)$ lying below $M$ (for the first octant; definitions for other octants are straightforward). We denote this discrete estimator by $\mathcal{T}_{M}^{\mathrm{ms}}$. We shall prove in Theorem 3 that this estimator is multigrid convergent with average convergence speed of $\mathcal{O}\left(h^{\frac{2}{3}}\right)$. As a corollary, the FeschetTougne tangent estimator [6], which uses the most centered maximal segment around $M$, and the $\lambda$-MST estimator 12, which makes a convex combination of the directions of all maximal segments around $M$, are also multigrid convergent with same average speed.

Before proving this theorem, note first that the experimental evaluation of the $\lambda$-MST estimator, whose absolute error is displayed in Fig. [, left, suggests that its average convergence speed is indeed likely to be in $\mathcal{O}\left(h^{\frac{2}{3}}\right)$. Secondly, the average Minkowski length of maximal segments behave as the average length of the edges, that is in $\mathcal{O}\left(h^{\frac{2}{3}}\right)$ as shown on Fig. 14, right (the Minkowski length is $h$ times the digital length). Thirdly, every maximal segment contains a digital edge as stated in the following proposition whose proof can be found in [11]:

Proposition 4. On the border of a CDP $\Gamma$, any maximal segment, whose slope is some $\frac{p_{n}}{q_{n}}$, contains at least one digital edge that has either the same slope or its $n-1$-convergent $\frac{p_{n-1}}{q_{n-1}}$.

We may now state our main result, which relies also on Claim 2 . 
Theorem 3. Let $M$ be a point in the plane and let $\mathbb{F}_{c}^{3}(M)$ be the subset of shapes $S$ of $\mathbb{F}_{c}^{3}$ with $M \in \partial S$. The discrete estimator $\mathcal{T}_{M}^{\mathrm{ms}}$ at point $M$ is multigrid convergent toward the tangent direction $\theta_{M}$ for $\mathbb{F}_{c}^{3}(M)$ and Gauss digitization. Furthermore, its speed of convergence is on average $\mathcal{O}\left(h^{\frac{2}{3}}\right)$.

Proof. We take the same notations as in the proof of Proposition 3. We consider a maximal segment $M S$ below $M$ with slope $z_{n}$. According to Proposition 1 , it contains a digital edge $V V^{\prime}$ of slope $z_{n}$ or $z_{n-1}$, which may not be below $M$. Worst case is for a digital edge with slope $z_{n-1}$ and this is the one considered below. We have:

$$
\begin{aligned}
\left|\mathcal{T}_{M}^{\mathrm{ms}}(S)-\theta_{M}(S)\right| & \leq\left|z_{n}-f^{\prime}(0)\right| \\
& \leq\left|z_{n-1}-f^{\prime}(0)\right|+\left|z_{n}-z_{n-1}\right| \\
& \leq\left|z_{n-1}-f^{\prime}(x)\right|+\mathcal{O}(x)+\frac{1}{q_{n} q_{n-1}}
\end{aligned}
$$

using Taylor relation and Eq. (11) to get the last inequality.

If we choose some abscissa $x$ such that $(x, f(x))$ is above the edge $V V^{\prime}$, then the proof of Proposition 3 indicates that $\left|z_{n-1}-f^{\prime}(x)\right| \leq \mathcal{O}\left(h^{\frac{2}{3}}\right)$ on average. Since $(x, f(x))$ is above $M S$ too, $x$ cannot be greater than the length of $M S$, which is also some $\mathcal{O}\left(h^{\frac{2}{3}}\right)$ on average. At last, Claim 2 similarly provides $\frac{1}{q_{n} q_{n-1}}=\Theta\left(h^{\frac{2}{3}}\right)$. Summing all these bounds concludes the proof.

As an immediate corollary, the $\lambda$-MST estimator [12] and the Feschet-Tougne tangent estimator [6] have the same asymptotic behaviour.

\section{Conclusion}

We have studied properties of digital convex polygons and exhibited several new results (Proposition 1 and Proposition 1 ). We have also examined the asymptotic properties of digital edges on digitized convex shapes (Claim 2), which has led to a position estimator of average convergence speed $\mathcal{O}\left(h^{\frac{4}{3}}\right)$ (Proposition 2) and to a tangent direction estimator of average convergence speed $\mathcal{O}\left(h^{\frac{2}{3}}\right)$ (Proposition 3). At last we have shown the new bound of $\mathcal{O}\left(h^{\frac{2}{3}}\right)$ for the average convergence speed of tangent estimators based on maximal segments (Theorem 3), which matches experimental evaluation.

These results indicate that curvature estimators relying on surrounding DSS have most probably an error of $\mathcal{O}\left(h^{0}\right)$ : position $\equiv f(x)$ in $\mathcal{O}\left(h^{\frac{4}{3}}\right)$, tangent direction $\equiv f^{\prime}(x)$ in $\mathcal{O}\left(h^{\frac{2}{3}}\right)$, curvature $\equiv f^{\prime \prime}(x)$ probably in $\mathcal{O}(1)$ (uncertainty on tangent further divided by $\left.\mathcal{O}\left(h^{\frac{2}{3}}\right)\right)$. The problem of exhibiting a multigrid convergent curvature estimator is thus still open.

Another straightforward extension of this work would be to investigate the properties of discrete surfaces and estimators based on digital plane recognition. However since the problem of finding an enclosing polyhedron with a minimal number of 2-facets has been proven to be NP-hard (see [3), the problem would get much harder than the two dimensional case. 


\section{References}

1. A. Balog and I. Bárány. On the convex hull of the integer points in a disc. In $S C G$ '91: Proceedings of the seventh annual symposium on Computational geometry, pages 162-165. ACM Press, 1991.

2. J. Berstel and A. De Luca. Sturmian words, lyndon words and trees. Theoret. Comput. Sci., 178(1-2):171-203, 1997.

3. V.E. Brimkov. Discrete volume polyhedrization is np-hard. Research Report CITRTR-179, Centre for Image Technology and Robotics, University of Auckland, New Zealand, 2006.

4. D. Coeurjolly. Algorithmique et géométrie discrète pour la caractérisation des courbes et des surfaces. PhD thesis, Université Lumière Lyon 2, France, 2002.

5. F. de Vieilleville, J.-O. Lachaud, and F. Feschet. Maximal digital straight segments and convergence of discrete geometric estimators. In 14 th Scandinavian Conference on Image Analysis, LNCS 3540, pages 988-997. Springer-Verlag, 2005.

6. F. Feschet and L. Tougne. Optimal time computation of the tangent of a discrete curve: application to the curvature. In Discrete Geometry and Computer Imagery (DGCI), LNCS 1568, pages 31-40. Springer Verlag, 1999.

7. G. H. Hardy and E. M. Wright. An introduction to the theory of numbers. Oxford University Press, fourth edition, 1960.

8. M. N. Huxley. Exponential sums and lattice points. Proc. of London Math.Soc., 60:471-502, 1990.

9. R. Klette and A. Rosenfeld. Digital Geometry - Geometric Methods for Digital Picture Analysis. Morgan Kaufmann, San Francisco, 2004.

10. R. Klette and J. Žunić. Multigrid convergence of calculated features in image analysis. Journal of Mathematical Imaging and Vision, 13:173-191, 2000.

11. J.-O. Lachaud and F. de Vieilleville. Convex shapes and convergence speed of discrete tangent estimators. Research Report RR-1398-06, LaBRI, University Bordeaux 1, Talence, France, 2006.

12. J.-O. Lachaud, A. Vialard, and F. de Vieilleville. Analysis and comparative evaluation of discrete tangent estimators. In E. Andrès, G. Damiand, and P. Lienhardt, editors, Proc. Int. Conf. Discrete Geometry for Computer Imagery, LNCS 3429, pages 240-251. Springer-Verlag, 2005.

13. J.-O. Lachaud, A. Vialard, and F. de Vieilleville. Fast, accurate and convergent tangent estimation on digital contours. Image Vis. Comput., 2006. In revision.

14. H. Reiter-Doerksen and I. Debled-Rennesson. Convex and concave parts of digital curves. In Dagstuhl Seminar "Geometric Properties from Incomplete Data", March 2004.

15. J.-P. Réveillès. Géométrie discrète, calcul en nombres entiers et algorithmique. Thèse d'etat, Université Louis Pasteur, Strasbourg, 1991.

16. F. Sloboda, B. Zaťko, and J. Stoer. On approximation of planar one-dimensional continua. In R. Klette, A. Rosenfeld, and F. Sloboda, editors, Advances in Digital and Computational Geometry, pages 113-160, 1998.

17. A. Vialard. Geometrical parameters extraction from discrete paths. In Proc. DGCI'96, volume 1176 of LNCS, pages 24-35. Springer, 1996.

18. K. Voss. Discrete Images, Objects, and Functions in $\mathbb{Z}^{n}$. Springer-Verlag, 1993. 